\title{
La Inactividad Física en Etapas Tempranas del Desarrollo Disminuye la Expresión de Akt en el Músculo Sóleo de Ratas
}

\author{
Akt Expression is Diminished by Physical Inactivity in \\ Early Stages of Development in Soleus Muscle of Rats
}

\author{
"José L. Márquez; **Sandro M. Hirabara; **Jarlei Fiamoncini; "*Elaine Hatanaka; \\ ***Tatiana C. Alba-Loureiro; **Thais M. de Lima-Salgado; ${ }^{* *}$ Rui Curi \& ${ }^{* * *}$ Luis A. Salazar
}

\begin{abstract}
MÁRQUEZ, J. L.; HIRABARA, S. M.; FIAMONCINI, F.; HATANAKA, E.; ALBA-LOUREIRO, T. C.; LIMA-SALGADO, T. M.; CURI, R. \& SALAZAR, L. A. La inactividad física en etapas tempranas del desarrollo disminuye la expresión de Akt en el músculo sóleo de ratas. Int. J. Morphol., 29(1):256-267, 2011.
\end{abstract}

RESUMEN: El Síndrome Metabólico corresponde a una serie de trastornos relacionados con obesidad e inactividad física. Poco se conoce respecto de la falta de ejercicio, en estadios tempranos del desarrollo, en la susceptibilidad a un fenotipo insulinoresistente inducido por una dieta alta en grasas. Akt juega un rol clave en la síntesis de proteínas y el transporte de glucosa en el músculo esquelético y ha mostrado ser regulada por la actividad muscular. El objetivo del presente estudio fue determinar el efecto de la inactividad física temprana sobre el crecimiento muscular y la susceptibilidad de adquirir un fenotipo diabético y evaluar su relación con la expresión de Akt. Cuarenta ratas Wistar fueron distribuidas en 2 grupos (Grupos Control, Std) y Restricción de movimiento (RM). Entre los días postnatal 23 y 70 los animales del grupo RM fueron alojados en pequeñas jaulas que no permitían una actividad motora relevante. A partir del día postnatal 71 y hasta el día 102, 10 ratas de cada grupo fueron alimentadas con una Dieta Alta en Grasas (RM-DAG y StdDAG). No se observaron diferencias en el peso corporal total pero DAG generó un significativo incremento en la grasa epididimal. RM generó una disminución significativa en el peso de los músculos sóleo. La captación de glucosa estimulada por insulina fue menor en el grupo RM-DAG. Los niveles de proteína Akt fueron menores en los grupos RM. El análisis de PCR a tiempo real mostró que la restricción de movimiento disminuyó los niveles de ARNm de AKT1 en el músculo sóleo, independiente de la dieta administrada. Estos hallazgos sugieren que la inactividad física temprana limita el crecimiento muscular y contribuye en la instauración un fenotipo insulino resistente, lo cual puede ser en parte explicado por una desregulación en la expresión de Akt.

PALABRAS CLAVE: Akt; Inactividad física; Insulinoresistencia.

\section{INTRODUCCIÓN}

El síndrome metabólico corresponde a un conjunto de anormalidades que involucran la presencia de obesidad abdominal, insulino resistencia y dislipidemia entre otras alteraciones y se asocia con otras comorbilidades como un estado protrombótico, proinflamatorio y desórdenes reproductivos (Cornier et al., 2008). Factores genéticos y ambientales, que incluyen dieta alta en grasa e inactividad física, contribuyen en diversas proporciones a su instauración (Potenza \& Mechanick, 2009) de tal modo que un bajo nivel de actividad física, en etapas tempranas del desarro- llo, puede condicionar críticamente la susceptibilidad a estos trastornos.

La expresión de muchos genes y proteínas involucradas en el metabolismo de la glucosa y la señalización por insulina han mostrado ser regulados por grasas (Silveira et al., 2008) y por ejercicio (Pauli et al., 2009), tanto en modelos humanos como múridos, sin embargo, ningún estudio ha utilizado un modelo de restricción de movimiento, por limitación de espacio, durante etapas críticas

\footnotetext{
" Programa de Doctorado en Ciencias, mención Biología Celular y Molecular Aplicada, Universidad de La Frontera, Temuco, Chile; Programa de Doutorado em Fisiologia Humana, Instituto de Ciências Biomédicas, Universidade de São Paulo, Brasil; Laboratorio de Biología Molecular y Farmacogenética, Depto. de Ciencias Básicas, Universidad de La Frontera, Temuco, Chile; Departamento de Kinesiología, Universidad Católica del Maule, Talca, Chile.

** Laboratório de Fisiologia Celular, Departamento de Fisiologia e Biofísica, Instituto de Ciências Biomédicas, ICB, Universidade de São Paulo, Brasil.

*** Laboratorio de Biología Molecular y Farmacogenética, Departamento de Ciencias Básicas, Facultad de Medicina, Universidad de La Frontera, Temuco, Chile; Núcleo de Desarrollo Científico - Tecnológico en Biorecursos, Universidad de La Frontera (BIOREN-UFRO), Temuco, Chile.
} 
del desarrollo postnatal para evaluar la respuesta adaptativa de alguno de ellos frente a una dieta alta en grasas, administrada durante la vida adulta.

En este contexto, nosotros proponemos a Akt como un regulador clave cuya desregulación puede estar relacionada con mayor ganancia de peso, menor proporción de masa magra y una alterada captación de glucosa en el músculo esquelético, jugando un rol significativo en el origen del síndrome metabólico.

Akt, también conocida como PKB es una proteína serina/treonina kinasa que juega un rol crítico en la transducción de señales inducidas por insulina, siendo un blanco corriente abajo de PI3k (Taniguchi et al., 2006). Esta proteína presenta en mamíferos, tres isoformas, Akt1, Akt2 y Akt3, mientras las dos primeras responden intensamente a insulina, la tercera lo hace débilmente. Junto con su participación en la captación de glucosa por insulina, Akt ha mostrado jugar un rol critico en el crecimiento de ratones, tanto in útero, como luego del nacimiento (Cho et al., 2001).

La expresión de proteína Akt puede ser regulada por muchos factores ambientales y fisiológicos. La exposición crónica a especies reactivas de oxígeno ha mostrado regular a la baja la expresión de Akt in vitro e in vivo (Martin et al., 2002) y Crossland et al., (2008) han mostrado que los lipopolisacáridos inducen una reducción de Akt en conjunto con una mayor degradación proteica muscular. Por otro lado, Bodine et al., (2001) ha mostrado un incremento en la expresión de Akt durante la hipertrofia muscular.

En músculos esqueléticos insulino resistentes, Akt ha mostrado generalmente estar reducida y un incremento en su expresión se ha asociado con una disminución de esta condición (Wu et al., 2011). Paciente obesos con hiperglicemia tienen menor expresión de proteína Akt2 muscular y la normalización de la glicemia luego de una terapia insulínica logra incrementar los niveles basales de la proteína (Gosmanov et al., 2004). Por otra parte, la administración aguda de tiazolidinedionas ha mostrado incrementar los niveles de Akt muscular (Planavila et al., 2005).
La insulino resistencia inducida por dieta alta en grasa, por su parte, ha mostrado disminuir la expresión muscular de Akt1, condición que es revertida por el ejercicio físico (Lessard et al., 2007). De esta forma, un incremento en la expresión muscular de Akt o una disminución en su degradación pueden ayudar a aminorar la insulino resistencia.

Así, la presente investigación fue diseñada para evaluar los efectos de la inactividad física, durante un periodo postnatal temprano, sobre el crecimiento muscular y la instauración de un fenotipo insulino resistente en presencia de una dieta alta en grasas.

\section{MATERIAL Y MÉTODO}

Animales. Cuarenta ratas macho Wistar recién desmamados (peso inicial, 49,5 $\pm 5,21 \mathrm{~g}$ ) fueron obtenidas del Bioterio del Instituto de Ciencias Biomédicas de la Universidad de São Paulo, Brasil. Los experimentos fueron realizados siguiendo los cuidados detallados en The Principles for the Care and Use of Animals (Institute for Laboratory Animal Research, 1996).

Los animales fueron alojados en una sala con un ciclo invertido de 12 horas de luz/oscuridad, bajo condiciones estandarizadas de temperatura y humedad. Inicialmente los animales fueron distribuidos aleatoriamente en dos grupos experimentales (RM: Restricción de Movimiento y Std: Grupo Control o condiciones estándar) y alojados en grupos de 5. Para el grupo Std, las jaulas presentaban una superficie de $1200 \mathrm{~cm}^{2}$, mientras que las ratas del grupo RM, la superficie de la jaula fue modificada desde 250 hasta 500 $\mathrm{cm}^{2}$ (día postnatal 23 al día posnatal 70), de tal forma que los animales se pudiesen mover para obtener alimento y agua pero que no dispusieran de espacio suficiente para otra actividad motora relevante. Durante este periodo las ratas fueron alimentadas con una dieta Estándar (Nuvilab CR1).

El día postnatal 70, las ratas de cada grupo fueron distribuidas aleatoriamente en dos subgrupos, conformando

Tabla I. Características de las dietas utilizadas durante el estudio.

\begin{tabular}{|c|c|c|c|}
\hline & $\begin{array}{l}\text { Dieta de laboratorio estándar } \\
\text { (Nuvilab CR1) }\end{array}$ & $\begin{array}{l}\text { Dieta control } \\
\text { (AIN-93M) }\end{array}$ & $\begin{array}{c}\text { Dieta alta en grasa } \\
\text { (AIN-93M hiperlipídica) }\end{array}$ \\
\hline Proteínas & $22 \%$ & $13,06 \%$ (13,4\% de la energía) & $14 \%$ (9,8 de la energía) \\
\hline Grasas & $4 \%$ & $4 \% \quad(9,7 \%$ de la energía $)$ & $35,5 \%$ (55,9 de la energía) \\
\hline Carbohidratos & $62 \%$ & $73,8 \% \quad(77,1 \%$ de la energía $)$ & $49 \%(34,3 \%$ de la energía $)$ \\
\hline Fibra & $8 \%$ & $5 \%$ & $1,5 \%$ \\
\hline Energía (kcal/g) & 3,72 & 3,79 & 5,715 \\
\hline
\end{tabular}


MÁRQUEZ, J. L.; HIRABARA, S. M.; FIAMONCINI, F.; HATANAKA, E.; ALBA-LOUREIRO, T. C.; LIMA-SALGADO, T. M.; CURI, R. \& SALAZAR, L. A. La inactividad física en etapas tempranas del desarrollo disminuye la expresión de Akt en el músculo sóleo de ratas. Int. J. Morphol., 29(1):256-267, 2011.

finalmente cuatro, denominados: Std-DC; Std-DAG; RMDC y RM-DAG. Las ratas de los subgrupos DC fueron alimentadas con una dieta control AIN-93M mientras que los animales de los subgrupos DAG fueron alimentadas con una dieta alta en grasas (las características de las dietas se muestran en la Tabla I). Un máximo de 5 animales fue alojado en cada jaula de $1200 \mathrm{~cm}^{2}$ y a partir de este momento no existieron diferencias en la superficie disponible para el desplazamiento de las ratas en los diferentes grupos. El día postnatal 103 los animales fueron sacrificados, la sangre y los músculos sóleo fueron obtenidos, separados en tres bandas y utilizados para evaluar la captación de glucosa o almacenados para los análisis fisiológicos y moleculares posteriores. Junto con esto se registró el peso paquete graso epididimal y el peso corporal total.

Mediciones de glucosa e insulina en plasma. Las ratas fueron sacrificadas por decapitación y la sangre fue colectada en tubos heparinizados. El plasma fue obtenido por centrifugación a $600 \mathrm{x}$ g por 20 minutos a $4^{\circ} \mathrm{C}$ y luego fue almacenada en alícuotas a $-80^{\circ} \mathrm{C}$ hasta el momento de realizar las determinaciones. Las concentraciones de glucosa fueron medidas utilizando el kit "Laborlab glicose test kit" (Laborlab, Sao Paulo, Brasil) y los niveles de insulina por Radioinmunoensayo con doble anticuerpo (Pharmacia, Uppsala, Suecia).

Captación de glucosa inducida por insulina en músculo aislado. Cada músculo sóleo fue aislado y dividido en tres porciones, la porción central fue inmediatamente congelada en nitrógeno líquido para su posterior análisis y las porciones laterales (25-35 mg) fueron usadas para los experimentos de captación de glucosa. Las bandas de músculo fueron preincubadas por 30 minutos en Tampón Bicarbonato Ringer-Krebs (pH 7,4) que contenía 5,5 mM de glucosa y $1,5 \%(\mathrm{p} / \mathrm{v})$ de albúmina de suero bovino. Posteriormente las muestras fueron transferidas a viales que contenían un medio idéntico al de preincubación más $0,1 \mu \mathrm{Ci}$ de 2-DeoxyD- $\left[2,6-{ }^{3} \mathrm{H}\right]$ glucosa/mL e insulina. La incubación fue realizada por 1 hora en ausencia o presencia de 100, 1,000 o $10,000 \mu \mathrm{U} / \mathrm{mL}$ de insulina. Todos los tampones fueron gasificados continuamente con $95 \% \mathrm{O}_{2} / 5 \% \mathrm{CO}_{2}$. Una vez terminada la incubación, los músculos fueron lavados en tampón helado, secados rápidamente y congelados en nitrógeno líquido. Para el procesamiento, los músculos fueron hidrolisados en 0,5 mL de KOH 1 Molar, $150 \mu \mathrm{L}$ del extracto fueron transferidos a viales de conteo a los cuales se agregó $1,5 \mathrm{~mL}$ de líquido de centelleo. La radioactividad para $\mathrm{H}^{3}$ fue cuantificada por centelleografía líquida en un equipo Beckman LS 7500. La tasa de transporte del análogo de glucosa no metabolizable 2-Deoxy-D-[2,6- $\left.{ }^{3} \mathrm{H}\right]$ glucosa, fue medida como se describe previamente (Crettaz et al., 1980; Challiss et al., 1986). La acumulación de 2-Deoxy-D-[2,6-
3H] glucosa en el espacio intersticial fue corregida a partir de datos de experimentos previos con el inhibidor inespecífico del transporte de glucosa, floretina.

Western blotting. Se obtuvieron proteínas totales a partir de las bandas centrales de músculo sóleo no utilizadas para evaluar la captación de glucosa. El tejido fue homogenizado en $500 \mu \mathrm{L}$ de tampón de extracción helado $(100 \mathrm{mM}$ Tris, pH 7.5; 10mM EDTA; $10 \%$ SDS; $100 \mathrm{mM} \mathrm{NaF} ; 10 \mathrm{mM}$ pirofosfato de sodio y $10 \mathrm{mM}$ de ortovanadato de sodio) a $4^{\circ} \mathrm{C}$, por 30 segundos. Se agregó Tritón X-100 al 1\% y se incubó por 30 minutos a $4^{\circ} \mathrm{C}$. Las muestras fueron luego centrifugadas a $13,000 \mathrm{x}$ g por 20 minutos a $4^{\circ} \mathrm{C}$. Alicuotas del sobrenadante fueron utilizadas para medir el contenido total de proteínas según el procedimiento descrito por Bradford (1976). Cantidades idénticas de proteínas de cada muestra $(40 \mu \mathrm{g})$ fueron separadas por electroforesis en un gel de poliacrilamida-SDS al 10\%. El procedimiento de Western Blot se realizó utilizando el método descrito por Towbin et al. (1979). Las proteínas del gel fueron transferidas a membranas de PVDF a $140 \mathrm{~V}$ por 2 horas. Para evitar la unión inespecífica de los anticuerpos, las membranas fueron incubadas en una solución basal (10mM Tris, $\mathrm{pH} 7,5$; $150 \mathrm{mM} \mathrm{NaCl} ; 0,05 \%$ Tween 20) que contenía leche desnatada al 5\%, a temperatura ambiente por 2 horas. Las membranas fueron luego lavadas en solución basal tres veces de 10 minutos cada una y luego incubadas con anticuerpo anti-Akt (Cell Signalling, dilución 1:1000) en una solución basal que contenía $3 \%$ de leche desnatada, a $4^{\circ} \mathrm{C}$ toda la noche. Las membranas fueron luego lavadas nuevamente (3 veces de 10 minutos) e incubadas con un anticuerpo secundario anti-IgG conjugado con peroxidada de rábano en una solución basal que contenía leche desnatada al 1\%, a temperatura ambiente por 2 horas. A seguir las membranas fueron nuevamente lavadas e incubadas en un sustrato quimioluminiscente (ECL Western Blotting System) por 1 minuto e inmediatamente expuestos a una película de rayos-X. Las películas fueron luego procesadas de manera convencional. La carga de proteínas fue controlada utilizando las imágenes obtenidas del teñido de las membranas con rojo de Ponceau. Adicionalmente las bandas observadas en la película autorradiográfica fueron normalizadas contra aquellas generadas por el uso del anticuerpo $\beta$-actina (Cell Signalling, dilución 1:1000).

Extracción de RNA. La extracción de RNA desde tejido muscular de sóleo previamente congelado fue obtenido por el método de extracción de guanidinio isotiocianato usando el reactivo Trizol ${ }^{\circledR}$ (Invitrogen, California, USA). Brevemente, el tejido fue homogenizado en $1 \mathrm{~mL}$ de Trizol ${ }^{\circledR}$, después de 5 minutos de incubación a temperatura ambiente se agregó $200 \mu \mathrm{L}$ de cloroformo y los tubos fueron centrifugados a $12.000 \mathrm{x} \mathrm{g}$. La fase acuosa fue transferida a 
MÁRQUEZ, J. L.; HIRABARA, S. M.; FIAMONCINI, F.; HATANAKA, E.; ALBA-LOUREIRO, T. C.; LIMA-SALGADO, T. M.; CURI, R. \& SALAZAR, L. A. La inactividad física en etapas tempranas del desarrollo disminuye la expresión de Akt en el músculo sóleo de ratas. Int. J. Morphol., 29(1):256-267, 2011.

otro tubo que contenía isopropanol helado y el RNA fue precipitado por centrifugación (12.000 x g). El pellet fue lavado con etanol, nuevamente centrifugado y secado a temperatura ambiente. El RNA fue luego resuspendido en agua libre de RNAsas, alicuotado y almacenado a $-80^{\circ} \mathrm{C}$. El RNA fue cuantificado por medición de la absorbancia a $260 \mathrm{~nm}$. La pureza del RNA fue evaluada por la relación 260/280 $\mathrm{nm}$ y por electroforesis en un gel de agarosa al $1 \%$ teñido con bromuro de etidio $(5 \mu \mathrm{g} / \mathrm{mL})$.

Transcripción reversa. $2 \mu \mathrm{g}$ de RNA total fueron tratados con $1 \mathrm{U}$ de DNasa I durante 25 minutos a $25^{\circ} \mathrm{C}$ y posteriormente se inactivó la enzima con EDTA (2,5 mM). A seguir, el cDNA fue sintetizado en $20 \mu \mathrm{L}$ de medio que contenía 10 mM de cada dNTP, $10 \mathrm{mM}$ de DTT y 200 unidades de superscriptTM II Rnase $\mathrm{H}^{-}$, a $42^{\circ} \mathrm{C}$ por 50 minutos. El cDNA fue almacenado a $-20^{\circ} \mathrm{C}$ hasta la realización de la PCR a tiempo real.

PCR a tiempo real. La expresión de Akt1 fue evaluada por PCR a tiempo real utilizando un equipo ROTOR GENE 3000 (Corbett Research, Mortlake, Australia). Para la realización de la PCR a tiempo real se utilizó $1 \mu \mathrm{L}$ de cDNA en un volumen final de $25 \mu \mathrm{L}$, el que contenía $100 \mathrm{mM}$ of dNTPs, buffer de reacción 10x (10 mM Tris- $\mathrm{HCl}, 50 \mathrm{mM} \mathrm{KCl,} 2$ $\mathrm{mM} \mathrm{MgCl} 2$ ), $1 \mathrm{U}$ de Taq DNA polimerasa (Invitrogen Life Technologies, Rockville, MD, USA), 0,1 mM de cada primer (forward y reverse) y SYBR GREEN (Invitrogen, Carlsbad, CA, EUA). Las muestras fueron corridas en dos ocasiones separadas con un ciclo inicial a $94^{\circ} \mathrm{C}$ por $5 \mathrm{mi}-$ nutos, seguidos inmediatamente por 35 ciclos $\left(94^{\circ} \mathrm{C}\right.$ por 30 segundos de denaturación y $72^{\circ} \mathrm{C}$ por 60 segundos de extensión, la temperatura del ciclo de annealing varió dependiendo de la Tm de cada set de primers y tuvo una duración de 60 segundos). La fluorescencia resultante de la incorporación de SYBR GREEN en la doble hebra de DNA, producida durante la reacción de PCR fue registrada y los datos emitidos fueron cuantificados utilizando el valor de ciclo umbral (threshold cycle, CT). Las lecturas de CT obtenidas fueron representadas en una escala logarítmica. Una curva de disociación de melting, generada por el propio instrumento sirvió para confirmar que un solo producto estaba presente. Cada PCR incluyó posillos controles sin templado (en triplicado) agregando en su lugar agua libre de RNAsas a los pocillos de reacción. Los primers específicos fueron diseñados utilizando el software PRIMER3. La cuantificación de la expresión génica fue realizada según describe Liu \& Saint (2002), utilizando el gen $\beta$-2-Microglobulina $(\beta-2 \mathrm{M})$ como control interno. La eficiencia de amplificación fue calculada como describe Ramakers et al. (2003). La secuencia de los primers utilizados fueron las siguientes: AKT1 forward, 5'-TGG ACT CAAACG GCA GGA GG-3'; AKT1 reverse, 5'-ACAAAG
CAG AGG CGG TCG TG-3' y $\beta 2 \mathrm{M}$ forward, 5'-CTC AGT TCC ACC CAC CTC AG-3'; $\beta 2 \mathrm{M}$ reverse, 5'-GCA AGC ATA TAC ATC GGT CTC G-3'.

Análisis estadístico. Las diferencias entre los grupos fueron evaluadas utilizando ANOVA y el test-t para muestras no pareadas. ANOVA fue utilizada para realizar las comparaciones entre grupos seguida por el análisis post-hoc mediante el test de Tukey para la comparación entre grupos. Se aceptó como significativo un valor $p<0,05$. Los datos se presentan como promedios \pm Error estándar

\section{RESULTADOS}

Peso corporal total e ingesta calórica. El crecimiento de los animales y la ingesta calórica fueron medidos por 47 días en las ratas RM y Std. Comparadas con el grupo Std, las ratas del grupo RM presentaron un menor peso al día postnatal $70(233,1 \pm 28,4$ vs $264,7 \pm 28,1 ; \mathrm{P}<0,05)$. Las diferencias fueron evidentes al día 39 de intervención (Fig. 1A). No se observaron diferencias en el consumo energético de ambos grupos durante los primeros 47 días de intervención, sin embargo, cuando la ingesta calórica fue analizada semanalmente, observamos que el grupo RM consumió más energía que el grupo Std en las semanas 3 y 4 $(\mathrm{P}<0,05)$ (Fig. 1B).

Las curvas de crecimiento fueron similares durante la intervención dietaria (día postnatal 71 al día post natal 103, datos no mostrados). Al día postnatal 103 el peso corporal total no fue significativamente diferente entre los grupos Std $(363,9 \pm 6,2$ g y 378,7 \pm 8,8 g para Std-DC y StdDAG respectivamente) y RM DAG $(339,9 \pm 10,2 \mathrm{~g})$, sin embargo el peso de las ratas del grupo RM DC (333,8 \pm $7,8 \mathrm{~g}$ ) fue más bajo que el grupo Std DAG $(\mathrm{P}<0,05)$ (Fig. $2 \mathrm{~A})$. Durante la intervención dietaria no se observó diferencias en la ingesta energética por grupo $(\mathrm{P}=0,13)$, sin embargo, durante la primera semana la ingesta del grupo RM-DAG fue mayor que la de los grupos Std-DAG y RMDC $(\mathrm{P}<0,05)$ (Fig. 2B).

Ganancia de peso, grasa epididimal y porcentaje de grasa. La ganancia de peso no fue diferente entre los grupos alimentados con DC, en tanto que la DAG generó un aumento significativo en la ganancia de peso en el grupo RMDAG respecto de RM-DC (113,9 \pm 7,5 g vs 84,8 \pm 4,1 g; $\mathrm{P}<0,05)$ y solo se observó una tendencia en el grupo StdDAG respecto de Std-DC $(99,0 \pm 9,4 \mathrm{~g}$ vs 74,8 \pm 7,4 g; $\mathrm{P}=0,07)$.

El peso del paquete graso epididimal no fue dife- 

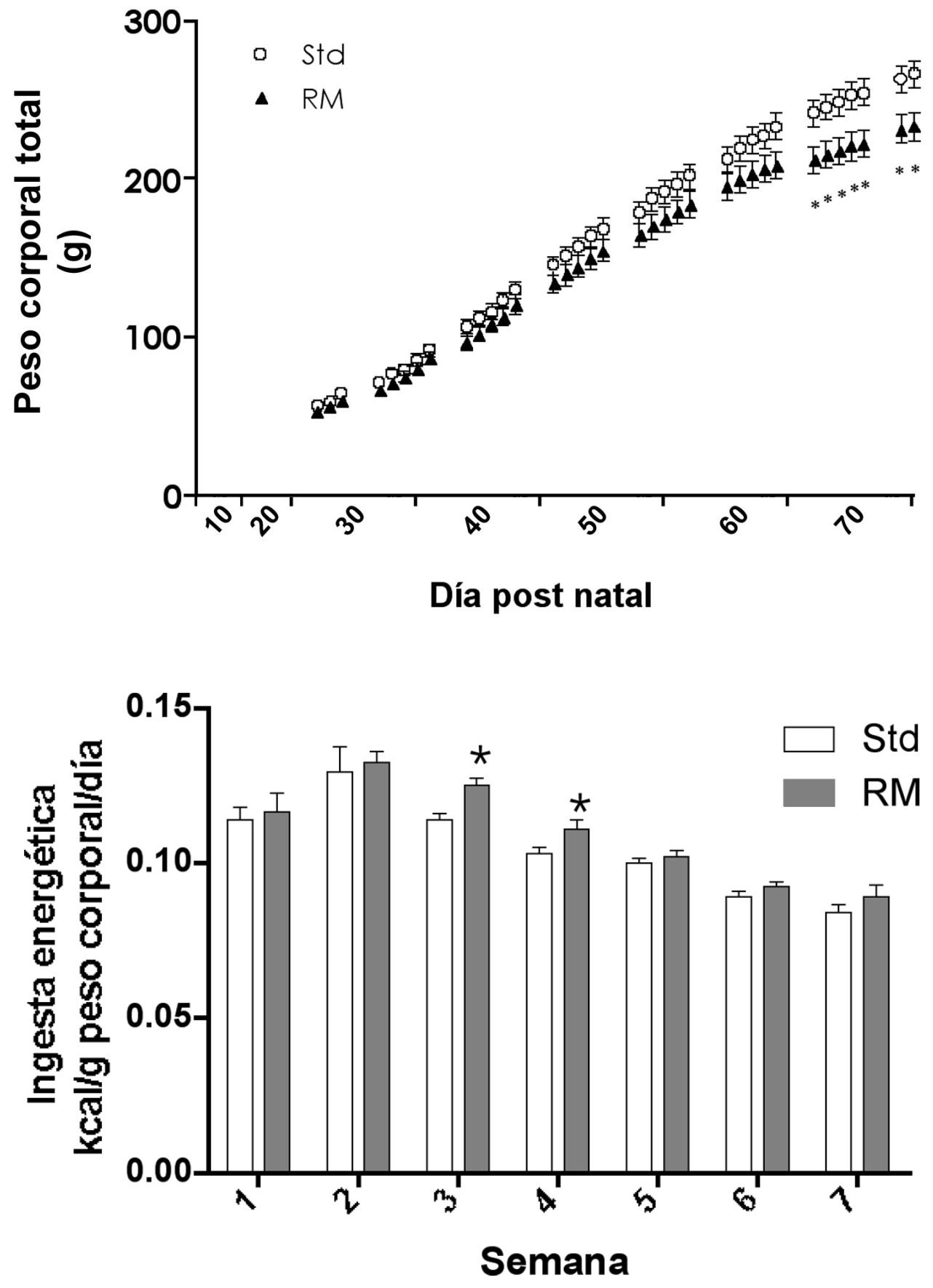

Fig. 1. Peso corporal acumulado (A) e ingesta energética (B) durante las primeras siete semanas de intervención. Los valores corresponden a la media \pm S.E.M, $\mathrm{n}=20$ por grupo. * $\mathrm{P}<0,05$.

rente entre los grupos alimentados con DC (4,3 \pm 0,2 g vs 5,0 \pm 0,4 g para RM-DC y StdDC respectivamente), sin embargo se observó un aumento significativo en el peso en el grupo RM-DAG $(5,8 \pm 0,5 \mathrm{~g})$ respecto del grupo $\mathrm{RM}-\mathrm{DC}(4,3 \pm 0,2 \mathrm{~g})(\mathrm{P}<0,05)$ y en el grupo Std-DAG $(6,6 \pm$ $0,4 \mathrm{~g})$ respecto del grupo Std-DC $(5,0 \pm 0,4 \mathrm{~g})$ $(\mathrm{P}<0,05)$. Cuando la grasa epididimal fue expresada en relación al peso corporal total los dos grupos alimentados con DAG (RM-DAG y Std-DAG) mostraron un incremento en la adiposidad respecto sus grupos control (1,6 \pm $0,09 \%$ vs $1,3 \pm 0,05 \%$ para RM-DAG vs RMDC y $1,7 \pm 0,07 \%$ vs $1,4 \pm 0,10 \%$ para StdDAG vs Std-DC; $\mathrm{P}<0,05$ ) (Tabla II).

Glucosa e insulina plasmática y captación de glucosa inducida por insulina. Los valores de glucosa e insulina de ayuno se muestran en la Tabla III. No se observaron diferencias entre los grupos al día postnatal 103. El transporte de glucosa inducido por $1,000 \mu \mathrm{U} /$ $\mathrm{mL}$ de insulina en el músculo sóleo de las ratas RM-DAG fue $19 \%$ menor que en el grupo RM-DC (4,75 土 0,9 vs $5,8 \pm 0,6 \mu \mathrm{mol} / \mathrm{h}$ por gramo de músculo; $\mathrm{P}<0,05)$. La reducción del $17 \%$ observada en Std-DAG respecto de Std-DC no resultó ser estadísticamente significativa $(\mathrm{P}=0,10)$. El transporte de glucosa en condiciones basales (ausencia de insulina) y en presencia de $100 \mu \mathrm{U} / \mathrm{mL}$ de insulina no mostró diferencias entre los grupos (Fig. 3). 

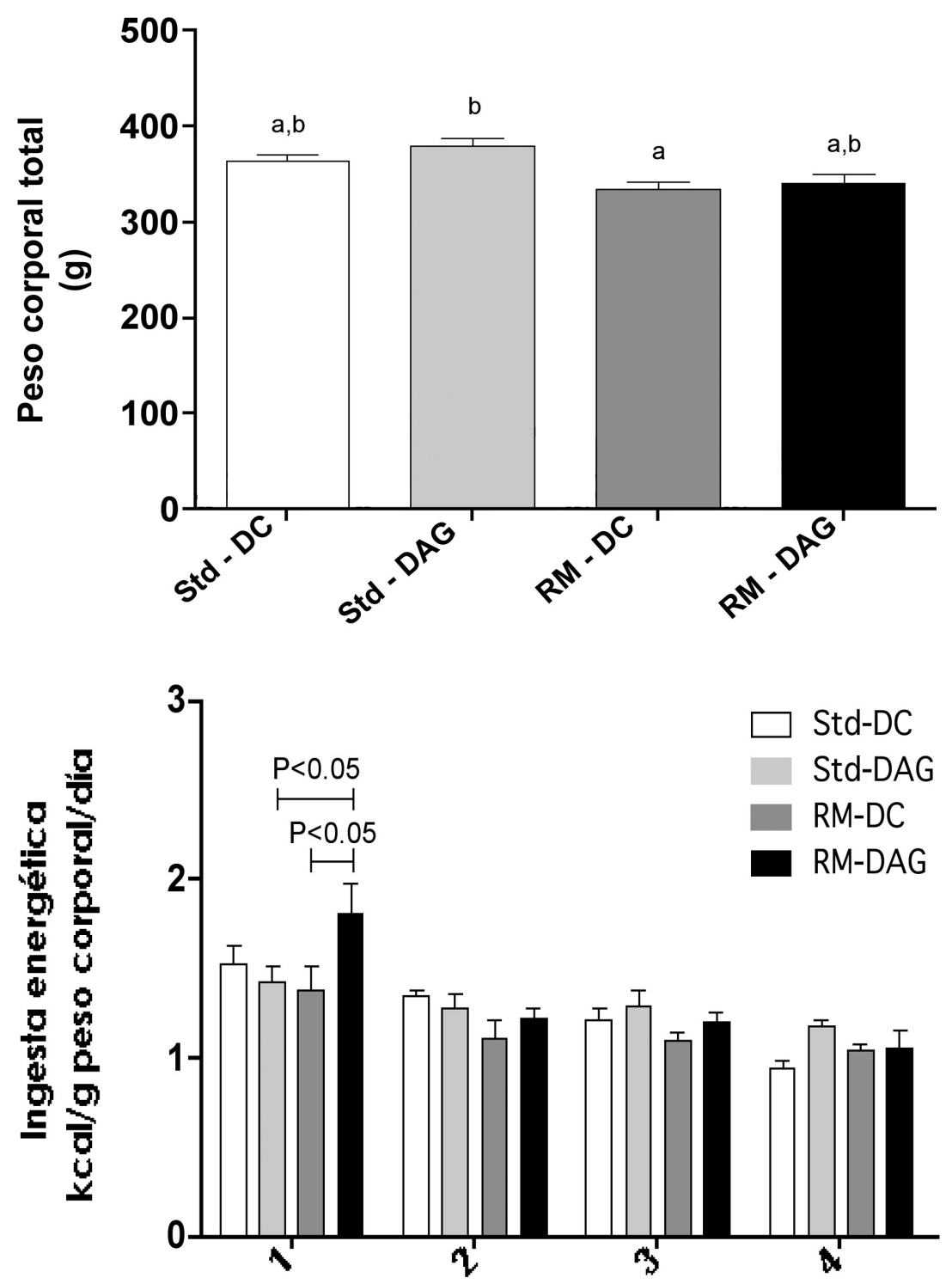

\section{Semana}

Fig. 2. Peso corporal final (A) e ingesta energética (B) de las ratas durante las cuatro semanas de intervención dietaria. Los valores corresponden a la media \pm S.E.M, $n=10$ por grupo.

${ }^{\mathrm{a}-\mathrm{b}}$ Letras distintas indican diferencias entre grupos, $\mathrm{P}<0,05$.

Tabla II. Ganancia de Peso durante la intervención dietaria, Grasa epididimal y Porcentaje de grasa ${ }^{1}$.

\begin{tabular}{lccc}
\hline Grupo & Ganancia de peso $(\mathrm{g})$ & Grasa epididimal $(\mathrm{GE}, \mathrm{g})$ & PC/GE \\
\hline Std-DC & $74,8 \pm 7,4$ & $5,0 \pm 0,4$ & $1,4 \pm 0,10$ \\
Std-DAG & $99,0 \pm 9,4$ & $6,6 \pm 0,4^{*}$ & $1,7 \pm 0,07^{*}$ \\
RM-DC & $84,8 \pm 4,1$ & $4,3 \pm 0,2$ & $1,3 \pm 0,05$ \\
RM-DAG & $113,9 \pm 7,5^{*}$ & $5,8 \pm 0,5^{*}$ & $1,6 \pm 0,09^{*}$ \\
\hline
\end{tabular}

${ }^{1}$ Los valores corresponden al promedio \pm SEM; $7-10$ ratas por grupo. * Representa diferencias significativas entre los grupos DAG y DC (p < 0.05). PC: Peso corporal total. 


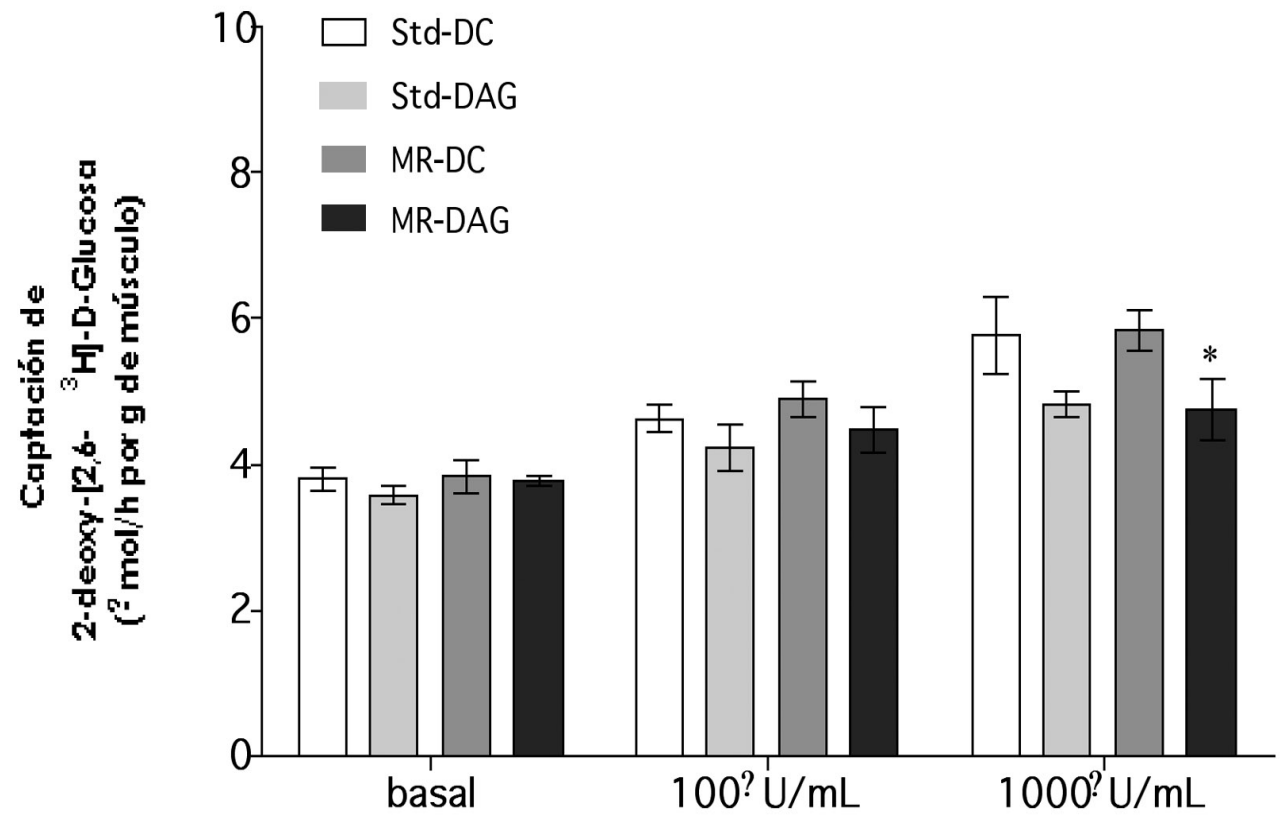

Fig. 3. Captación de 2-deoxy-D-[2,6- $\left.{ }^{3} \mathrm{H}\right]$ glucosa ( \pm S.E.M.) en músculo sóleo a diferentes concentraciones de insulina. Los valores corresponden a la media \pm S.E.M de 7-10 animales diferentes. ${ }^{*} \mathrm{P}<0,05$ vs RM-DC.

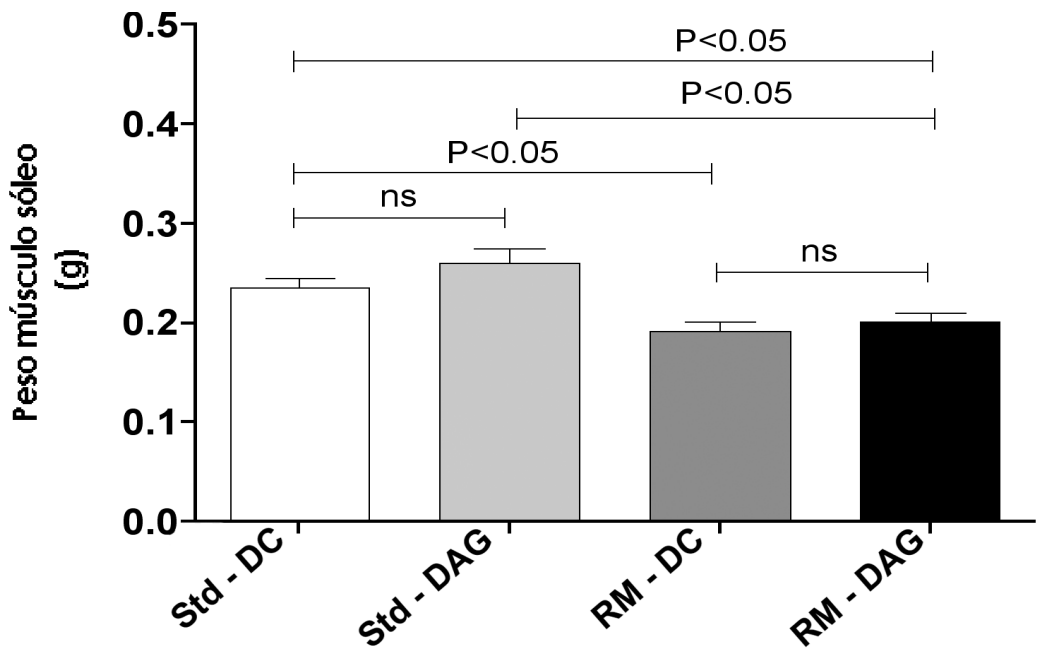

Fig. 4. Peso de ambos músculos soleo. Los valores corresponden a la media \pm S.E.M de 7-10 animales diferentes.

Tabla III. Glucosa e insulina plasmática y captación de glucosa mediada por insulina en músculo sóleo. 1Los valores corresponden al promedio \pm SEM; 7-10 ratas por grupo.

\begin{tabular}{cccc}
\hline Grupo & $\begin{array}{c}\text { Glucosa } \\
(\mathrm{mM})\end{array}$ & $\begin{array}{c}\text { Insulina } \\
(\mathrm{ng} / \mathrm{mL})\end{array}$ & $\begin{array}{l}\text { Captación de glucosa en } \\
\text { presencia de } 1000 \mu \mathrm{U} / \mathrm{mL} \\
\text { de insulina }(\mu \mathrm{mol} / \mathrm{h} \text { por } \\
\text { de músculo })\end{array}$ \\
\hline Std-DC & $6,8 \pm$ & $1,6 \pm$ & $5,8 \pm 1,3$ \\
Std-DAG & 0,3 & 0,16 & $4,8 \pm 0,5$ \\
& $0,8 \pm$ & $1,8 \pm$ & $5,8 \pm 0,6$ \\
RM-DC & $6,7 \pm$ & $1,4 \pm$ & 4,15 \\
& 0,3 & 0,05 & \\
RM-DAG & $6,6 \pm$ & $1,6 \pm$ & $4,9 \pm 0,9^{*}$ \\
& 0,4 & 0,16 & \\
\hline
\end{tabular}

"Representa diferencias significativas entre los grupos DAG y DC $(\mathrm{p}<0,05)$ 


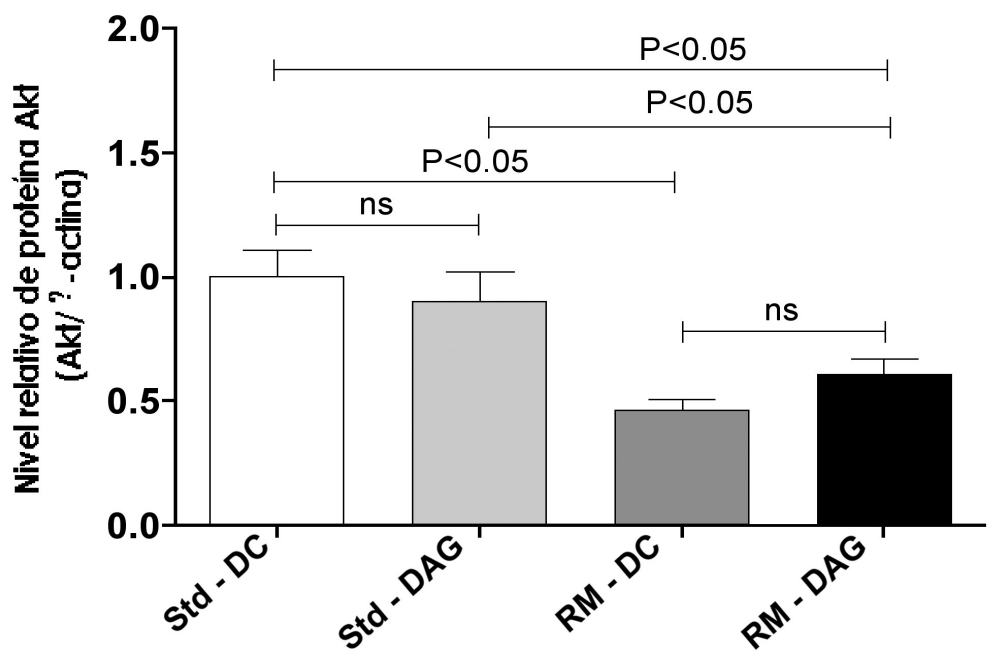

Fig. 5. Expresión proteica de Akt en músculo sóleo. Los valores corresponden a la media \pm S.E.M de 5-6 animales diferentes.

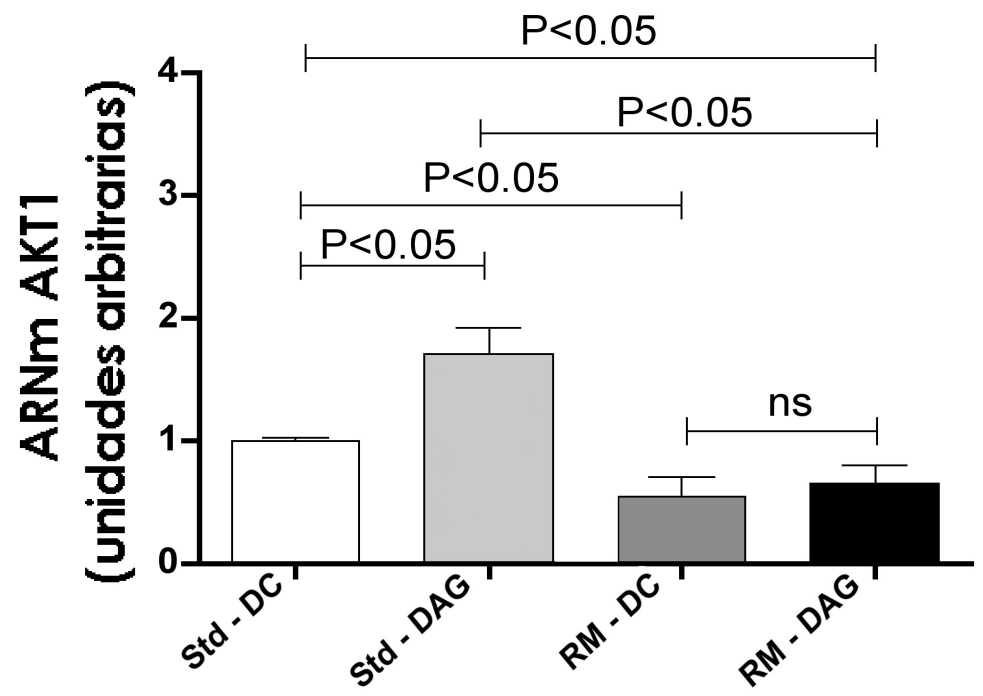

Fig. 6. Expresión génica de AKT1 en músculo sóleo. Los valores corresponden a la media \pm S.E.M de 5-8 animales diferentes.

Peso músculos sóleo. El peso de ambos músculos sóleo no fue diferente entre los subgrupos Std $(0,23 \pm 0,009 \mathrm{~g}$ vs $0,26 \pm 0,013 \mathrm{~g}$ para Std-DC y StdDAG respectivamente), ni RM $(0,19 \pm 0,009 \mathrm{~g}$ vs $0,20 \pm 0,010 \mathrm{~g}$ para RMDC y RM-DAG respectivamente). Sin embargo el peso de los músculos fue significativamente menor en los grupos RM respecto de los grupos Std $(\mathrm{P}<0,05)$ (Fig. 4).

Análisis de expresión proteica. Los niveles de proteína AKT no mostraron ser modificados por la dieta, sin embargo, se observó una disminución significativa en los niveles de expresión en los grupos RM respecto de los grupos Std $(\mathrm{P}<0,05)$, en una magnitud equivalente a 56 y $32 \%$ para DC y DAG respectivamente (Fig. 5).
Análisis de expresión génica. Los niveles de ARNm de AKT1 fueron más altos en el grupo Std-DAG $(\mathrm{P}<0,05)$, en tanto que la restricción de movimiento indujo una menor expresión del gen independiente de la dieta en una magnitud cercana al $35 \%$ $(\mathrm{P}<0,05)$ (Fig. 6).

\section{DISCUSIÓN}

Es ampliamente conocido que la actividad contráctil asociada con el ejercicio es un potente regulador de variadas funciones del músculo esquelético, incluyendo el transporte y metabolismo de glucosa y ácidos grasos. También se sabe que el ejercicio puede inducir transitorios cambios en la expresión génica y puede alterar las tasas de síntesis y degradación de proteínas musculares. En contraparte al ejercicio, la inactividad física se ha asociado con atrofia muscular, condición que ha mostrado estar relacionada con enfermedades como la diabetes, insuficiencia renal crónica y cáncer (Sacheck et al., 2007).

Dado que la inactividad física promueve la atrofia muscular, es posible que una disminución en la síntesis o un aumento en la degradación de proteínas musculares sean el nexo entre hábitos de vida sedentarios y el aumento en la prevalencia de las enfermedades crónicas no transmisibles, como Diabetes Tipo 2, aterosclerosis, hipertensión u obesidad (Booth et al., 2002).

En nuestro modelo de inactividad, restringimos el espacio disponible para realizar actividad física, durante un periodo en el cual el músculo esquelético se encuentra en desarrollo; así, esperábamos que esta intervención temprana generara adaptaciones que aumentaran la susceptibilidad, durante la vida adulta, frente a un estímulo que induce insulino resistencia como la dieta alta en grasas (Buettner et al., 2006; 2007).

Nuestros resultados muestran que la inactividad física durante un periodo de 
MÁRQUEZ, J. L.; HIRABARA, S. M.; FIAMONCINI, F.; HATANAKA, E.; ALBA-LOUREIRO, T. C.; LIMA-SALGADO, T. M.; CURI, R. \& SALAZAR, L. A. La inactividad física en etapas tempranas del desarrollo disminuye la expresión de Akt en el músculo sóleo de ratas. Int. J. Morphol., 29(1):256-267, 2011.

desarrollo de las ratas impactó directamente en el crecimiento de los animales, indicando que la inactividad física tiene efecto sobre la ganancia de peso en ratas Wistar juveniles. Una explicación para esta menor ganancia de peso lo constituye el hacinamiento de los animales durante su etapa de crecimiento. Yildiz et al. (2007) proponen que tal disminución estaría relacionada tanto con la falta de actividad como con la ingesta alimenticia, última situación que no ocurrió en nuestro estudio, puesto que las ratas con restricción de movimiento incluso tuvieron una mayor ingesta durante dos de las siete semanas de intervención. Otro factor que puede influenciar la menor ganacia de peso corresponde a cambios conductuales en los animales que incluyen agresión y tensión social (Brown \& Grunberg, 1995), sin embargo, esto parece ser más relevante en en ratas adultas que en ratas juveniles, en donde se observa frecuentemente una conducta de juego y no se exhibe una dominancia jerárquica o territorial (Arakawa, 2005). Así, en nuestro estudio la disminuida capacidad para ganar peso está probablemente relacionada con la limitada movilidad de los animales.

Durante la intervención dietaria de cuatro semanas, no se observaron diferencias significativas en la ingesta total, sin embargo durante la primera semana las ratas del subgrupo RM-DAG presentaron una mayor ingesta calórica que RM-DC y RM-DAG. Esta conducta hiperfágica inicial no fue mantenida en el tiempo. Al día postnatal 103, los animales alimentados con la dieta control no mostraron diferencias en el peso corporal total, lo mismo ocurrió entre los animales alimentados con dieta alta en grasas, las diferencias observadas entre el grupo Std-DAG y el grupo RMDC son atribuidas, a nuestro juicio, a un incremento en las diferencias de peso observadas al inicio de la intervención dietaria. Cuando se analizó la ganancia de peso en los grupos RM, se observó que DAG generó un aumento significativo en el peso corporal total respecto del grupo alimentado con DC, mientras que en los subgrupos Std, DAG no generó un cambio significativo. Esto indica que el efecto de DAG fue más fuerte en los animales previamente sedentarios. Cuando se compararon los pesos del paquete graso epididimal, se observaron aumentos significativos, inducidos por DAG en ambos grupos experimentales.

Estos datos, en conjunto, muestran que la inactividad física durante periodos de crecimiento, genera susceptibilidad a incrementar la adiposidad frente a una dieta alta en grasas, independiente de la ingesta calórica.

Estudios previos han asociado DAG con un fenotipo insulino resistente y es ampliamente aceptado que las grasas en la dieta alteran el metabolismo de la glucosa y las rutas de señalización molecular de la insulina, sin embargo, este efecto parece ser dependiente de la cepa de roedor uti- lizado, de la composición de la dieta y de la duración de la intervención (Buettner et al., 2006; 2007). En nuestro estudio, DAG contenía un 35,5\% (55,9\% de la energía) de grasas y fue administrada por 30 días. No observamos diferencias inducidas por la dieta en los niveles de glucosa ni insulina plasmática, indicando que esta forma de DAG no fue suficiente para inducir alteraciones en el metabolismo global de la glucosa; sin embargo, cuando evaluamos la captación de glucosa en músculo aislado, en presencia de 1,000 $\mu \mathrm{U} / \mathrm{mL}$ de insulina, observamos una reducción significativa en el grupo RM-DAG respecto de RM-DC, pero no en el grupo Std-DAG respecto de Std-DC. Estos datos indican que la inactividad física temprana predispone en mayor medida al efecto diabetogénico de la dieta alta en grasas, incrementando su efecto deletéreo.

Para evaluar el efecto de la restricción de movimiento sobre el crecimiento muscular, elegimos examinar un músculo de contracción lenta (músculo sóleo), por corresponder a un músculo que responde a modelos de atrofia por descarga (Dreyer et al., 2008) y que además es ampliamente utilizado en estudios de captación de glucosa (Sogaard et al., 2010). Nuestros datos indican una reducción significativa en el peso final de ambos músculoa sóleos al final de los experimentos (19\% y $23 \%$ para RM-DC y RM-DAG, respecto de sus controles). La reducción observada en el peso, independiente de la intervención dietaria de 4 semanas, indica que la restricción de movimiento temprana reduce el crecimiento de este músculo señalando a nuestro modelo de intervención como inductor de atrofia muscular.

Considerando que nuestro modelo presenta una disminución en el crecimiento muscular, asociado a un incremento en la susceptibilidad frente a una dieta alta en grasas en cuanto a la adiposidad e insulino resistencia, decidimos evaluar si estos cambios estaban relacionados con la expresión de una familia de proteínas clave en las rutas de señalización de atrofia y captación de glucosa (Boonyarom \& Inui, 2006; Dummler et al., 2006; Wu et al.).

Nuestros resultados muestran que la restricción temprana de movimiento genera, independiente de la dieta en la vida adulta, una disminución significativa en la expresión proteica de Akt, lo cual podría en parte explicar la reducción en el tamaño de los animales, la mayor ganancia de peso y adiposidad así como el menor crecimiento muscular y la mayor susceptibilidad a insulino resistencia en presencia de una dieta alta en grasas.

Tres diferentes genes codifican para tres proteínas Akt designadas como Akt1, Akt2 y Akt3. Akt1 y Akt2 son altamente expresadas en tejidos insulino sensibles, mientras que la expresión de Akt3 es baja en el músculo esquelé- 
tico (Masure et al., 1999). Estudios en modelos animales han mostrado que tanto Akt1 como Akt2 son necesarias para un óptimo desarrollo de la masa muscular (Frost \& Lang, 2007) en tanto que la ausencia de expresión de Akt 2 en ratones no reduce la captación de glucosa en el músculo sóleo (Cho et al.), lo que sitúa al gen de AKT1 como un posible blanco común de atrofia e insulino resistencia.

Cuando evaluamos la expresión génica de AKT1 observamos que DAG generó un aumento significativo en los niveles de ARNm lo cual podría representar una respuesta compensatoria primaria frente a la dieta alta en grasas. La falta de asociación con los niveles de expresión proteica de Akt total en este grupo sugiere la presencia de mecanismos postranscripcionales en su regulación. La disminución significativa en la expresión de AKT1 en los grupos RM, por su parte, se asociaron con la disminución en el contenido total de proteína Akt.

Drummond et al. (2008) Han mostrado que la inmovilidad inducida por sección de la médula espinal se asocia con disminución en los niveles de ARNm de AKT1 en músculo sóleo de ratas y lo mismo ha demostrado Dreyer et al. para el total de las proteínas Akt, concordando estos datos con los obtenidos en nuestro estudio.

Estos antecedentes, en conjunto, señalan a Akt1 como un potencial nexo entre la susceptibilidad a insulino resistencia y atrofia muscular en nuestro modelo de restricción de movimiento.

Los datos obtenidos en nuestro estudio, indican que la menor expresión de Akt en el músculo sóleo de ratas sometidas a restricción de movimiento por limitación de espacio, podría explicar, al menos en parte, la predisposición a insulino resistencia observada en presencia de una dieta elevada en grasas durante la vida adulta, en un proceso asociado a atrofia muscular.

\section{AGRADECIMIENTOS}

Los autores agradecen el apoyo técnico de José R. Mendonça y Érica P. Portioli. J. L. Márquez fue becario CONICYT-Chile.

MÁRQUEZ, J. L.; HIRABARA, S. M.; FIAMONCINI, F.; HATANAKA, E.; ALBA-LOUREIRO, T. C.; LIMA-SALGADO, T. M.; CURI, R. \& SALAZAR, L. A. Akt expression is diminished by physical inactivity in early stages of development in soleus muscle of rats. Int. J. Morphol., 29(1):256-267, 2011.

SUMMARY: Metabolic Syndrome is a group of conditions related to obesity and physical inactivity. Little is known about the role of physical inactivity, in early stages of development, in the susceptibility to insulin resistant phenotype induced by high fat diet. Akt plays a key role in protein synthesis and glucose transport in skeletal muscle and has been regulated by muscle activity. The objective of present study was to determine the effect of early physical inactivity on muscle growth and susceptibility to acquire a diabetic phenotype and to assess its relationship with Akt expression. Forty Wistar male rats were distributed in two groups (standard group, Std) and movement restriction (RM). Between days 23 and 70 after birth, RM group was kept in small cages that did not allow them to perform relevant motor activity. From day 71 to 102 after birth, 10 rats of each group were fed with hyperlipidic diet (groups Std-DAG and RMDAG). No differences were observed in total body weight although DAG increased epididymal fat pad weight. RM decreased significantly the soleus weight. Insulin-mediated glucose uptake was lower in RM-DAG group. Akt protein levels were lower in RM groups. Real time RT-PCR analysis showed that movement restriction decreased mRNA levels of AKT1 in soleus muscle, regardless of supplied diet. These findings suggest that early physical inactivity limits muscle's growth and contributes to instauration of insulin resistant phenotype, which can be partly explained by dysregulation of Akt expression.

\section{KEY WORDS: Akt; Physical inactivity; Insulin resistance.}

\section{REFERENCIAS}

Arakawa, H. Age dependent effects of space limitation and social tension on open-field behavior in male rats. Physiol. Behav., 84:429-36, 2005.

Bodine, S. C.; Stitt, T. N.; Gonzalez, M.; Kline, W. O.; Stover, G. L.; Bauerlein, R.; et al. Akt/mTOR pathway is a crucial regulator of skeletal muscle hypertrophy and can prevent muscle atrophy in vivo. Nat. Cell Biol., 3:1014-9, 2001.
Boonyarom, O. \& Inui, K. Atrophy and hypertrophy of skeletal muscles: structural and functional aspects. Acta Physiol., 188:77-89, 2006.

Booth, F. W.; Chakravarthy, M. V.; Gordon, S. E. \& Spangenburg, E. E. Waging war on physical inactivity: using modern molecular ammunition against an ancient enemy. J. Appl. Physiol., 93:3-30, 2002. 
MÁRQUEZ, J. L.; HIRABARA, S. M.; FIAMONCINI, F.; HATANAKA, E.; ALBA-LOUREIRO, T. C.; LIMA-SALGADO, T. M.; CURI, R. \& SALAZAR, L. A. La inactividad física en etapas tempranas del desarrollo disminuye la expresión de Akt en el músculo sóleo de ratas. Int. J. Morphol., 29(1):256-267, 2011.

Bradford, M. M. A rapid and sensitive method for the quantitation of microgram quantities of protein utilizing the principle of protein-dye binding. Anal. Biochem., $72: 248-54,1976$.

Brown, K. J. \& Grunberg, N. E. Effects of housing on male and female rats: Crowding stresses male but calm females. Physiol. Behav., 58:1085-9, 1995.

Buettner, R.; Parhofer, K. G.; Woenckhaus, M.; Wrede, C. E.; Kunz-Schughart, L. A.: Schölmerich, J.; et al. Defining high-fat-diet rat models: Metabolic and molecular effects of different fat types. J. Mol. Endocrinol., 36:485-501, 2006.

Buettner, R.; Schölmerich, J. \& Bollheimer, L. C. High-fat diets: modeling the metabolic disorders of human obesity in rodents. Obesity (Silver Spring)., 15:798-808, 2007.

Challiss, R. A.; Lozeman, F. J.; Leighton, B. \& Newsholme, E. A. Effects of the beta-adrenoceptor agonist isoprenaline on insulin-sensitivity in soleus muscle of the rat. Biochem. J., 233:377-81, 1986.

Cho, H.; Mu, J.; Kim, J. K.; Thorvaldsen, J. L.; Chu, Q.; Crenshaw, E. B.; et al. Insulin resistance and a diabetes mellitus-like syndrome in mice lacking the protein kinase Akt2 (PKB beta). Science, 292:1728-31, 2001.

Cornier, M. A.; Dabelea, D.; Hernandez, T. L.; Lindstrom, R. C.; Steig, A. J.; Stob, N. R.; et al. The metabolic syndrome. Endocr. Rev., 29:777-822, 2008.

Crettaz, M.; Prentki, M.; Zaninetti, D. \& Jeanrenaud, B. Insulin resistance in soleus muscle from obese Zucker rats. Involvement of several defective sites. Biochem. J., 186:525-34, 1980.

Crossland, H.; Constantin-Teodosiu, D.; Gardiner, S. M.; Constantin, D. \& Greenhaff, P. L. A potential role for Akt/FOXO signalling in both protein loss and the impairment of muscle carbohydrate oxidation during sepsis in rodent skeletal muscle. J. Physiol., 586:5589$600,2008$.

Dreyer, H. C.; Glynn, E. L.; Lujan, H. L.; Fry, C. S.; DiCarlo, S. E. \& Rasmussen, B. B. Chronic paraplegia-induced muscle atrophy downregulates the mTOR/S6K 1 signaling pathway. J. Appl. Physiol., 104:27-33, 2008.

Drummond, M. J.; Glynn, E. L.; Lujan, H. L.; Dicarlo, S. E. \& Rasmussen, B. B. Gene and protein expression associated with protein synthesis and breakdown in paraplegic skeletal muscle. Muscle Nerve, 37:505-13, 2008.

Dummler, B.; Tschopp, O.; Hynx, D.; Yang, Z. Z.; Dirnhofer, S. \& Hemmings, B. A. Life with a single isoform of Akt: mice lacking Akt 2 and Akt 3 are viable but display impaired glucose homeostasis and growth deficiencies. Mol. Cell Biol., 26:8042-51, 2006.

Frost, R. A. \& Lang, C. H. Protein kinase B/Akt: a nexus of growth factor and cytokine signaling in determining muscle mass. J. Appl. Physiol., 103:378-87, 2007.

Gosmanov, A. R.; Umpierrez, G. E.; Karabell, A. H.; Cuervo, R. \& Thomason, D. B. Impaired expression and insulin-stimulated phosphorylation of Akt-2 in muscle of obese patients with atypical diabetes. Am. J. Physiol. Endocrinol. Metab., 287:E8-E15, 2004.

Institute for Laboratory Animal Research. National Research Council. Guide for the Care and Use of Laboratory Animals. Washington DC, National Academy Press, 1996.

Lessard, S. J.; Rivas, D. A.; Chen, Z. P.; Bonen, A.; Febbraio, M. A.; Reeder, D. W.; et al. Tissue-specific effects of rosiglitazone and exercise in the treatment of lipidinduced insulin resistance. Diabetes, 56:1856-64, 2007.

Liu, W. \& Saint, D. A. Validation of a quantitative method for real time PCR kinetics. Biochem. Biophys. Res. Commun., 294:347-53, 2002.

Martin, D.; Salinas, M.; Fujita, N.; Tsuruo, T. \& Cuadrado, A. Ceramide and reactive oxygen species generated by $\mathrm{H} 2 \mathrm{O} 2$ induce caspase-3-independent degradation of Akt/ protein kinase B. J. Biol. Chem., 277:42943-52, 2002.

Masure, S.; Haefner, B.; Wesselink, J. J.; Hoefnagel, E.; Mortier, E.; Verhasselt, P.; et al. Molecular cloning, expression and characterization of the human serine/threonine kinase Akt3. Eur. J. Biochem., 265:353-60, 1999.

Pauli, J. R.; Cintra, D. E.; Souza, C. T. \& Ropelle, E. R. Novos mecanismos pelos quais o exercício físico melhora a resistência à insulina no músculo esquelético. Arq. Bras. Endocrinol. Metab., 53:399-408, 2009.

Planavila, A.; Alegret, M.; Sanchez, R. M.; Rodríguez-Calvo, R.; Laguna, J. C. \& Vazquez-Carrera, M. Increased Akt protein expression is associated with decreased ceramide content in skeletal muscle of troglitazonetreated mice. Biochem. Pharmacol., 69:1195-204, 2005. 
MÁRQUEZ, J. L.; HIRABARA, S. M.; FIAMONCINI, F.; HATANAKA, E.; ALBA-LOUREIRO, T. C.; LIMA-SALGADO, T. M.; CURI, R. \& SALAZAR, L. A. La inactividad física en etapas tempranas del desarrollo disminuye la expresión de Akt en el músculo sóleo de ratas. Int. J. Morphol., 29(1):256-267, 2011.

Potenza, M. V. \& Mechanick, J. I. The metabolic syndrome: definition, global impact, and pathophysiology. Nutr. Clin. Pract., 24:560-77, 2009.

Ramakers, C.; Ruijter, J. M.; Deprez, R. H. \& Moorman, A. F. Assumption-free analysis of quantitative real-time polymerase chain reaction (PCR) data. Neurosci. Lett., 339:62-6, 2003.

Sacheck, J. M.; Hyatt, J. P.; Raffaello, A.; Jagoe, R. T.; Roy, R. R.; Edgerton, V. R.; et al. Rapid disuse and denervation atrophy involve transcriptional changes similar to those of muscle wasting during systemic diseases. FASEB J., 21:140-55, 2007.

Silveira, L. R.; Fiamoncini, J.; Hirabara, S. M.; Procópio, J.; Cambiaghi, T. D.; Pinheiro, C. H.; et al. Updating the effects of fatty acids on skeletal muscle. J. Cell Physiol., 217:1-12, 2008.

Sogaard, P.; Szekeres, F.; Garcia-Roves, P. M.; Larsson, D.; Chibalin, A. V. \& Zierath, J. R. Spatial insulin signalling in isolated skeletal muscle preparations. J. Cell Biochem., 109:943-9, 2010.

Taniguchi, C. M.; Kondo, T.; Sajan, M.; Luo, J.; Bronson, R.; Asano, T.; et al. Divergent regulation of hepatic glucose and lipid metabolism by phosphoinositide 3kinase via Akt and PKClambda/zeta. Cell Metab., 3:34353, 2006.

Towbin, H.; Staehelin, T. \& Gordon, J. Electrophoretic transfer of proteins from polyacrylamide gels to nitrocellulose sheets: Procedure and some applications. Proc. Natl. Acad. Sci. USA, 76:4350-4, 1979.

Wu, M.; Falasca, M. \& Blough, E. R. Akt/protein kinase B in skeletal muscle physiology and pathology. J. Cell Physiol., 226:29-36, 2011.

Yildiz, A.; Hayirli, A.; Okumus, Z.; Kaynar, O. \& Kisa, F. Physiological profile of juvenile rats: Effects of cage size and cage density. Lab. Anim. (NY), 36:28-38, 2007.
Dirección para correspondencia:

Prof. Dr. Luis Antonio Salazar

Departamento de Ciencias Básicas,

Facultad de Medicina

Universidad de La Frontera

Av. Francisco Salazar 01145

Casilla 54-D

Temuco

CHILE

Tel.: +56 45592 895;

Fax: +56 45592832

Email: Isalazar@ufro.cl

Recibido : 14-10-2010

Aceptado: 22-11-2010 